\title{
Hypoxia Up-regulates HIF Expression While Suppressing Cell Growth and NOTCH Activity in Leukaemia Cells
}

\author{
MAI ITOH, YUKI OKUHASHI, YUSUKE TAKAHASHI, YURI SONODA, \\ SALWA MOHAMMAD, TATSUYA SAITO, ERIKA SHIRATORI and SHUJI TOHDA \\ Department of Laboratory Medicine, Tokyo Medical and Dental University, Tokyo, Japan
}

\begin{abstract}
Aim: To examine the influence of hypoxia on the in vitro growth of leukaemia cells and the activity of signalling proteins to better understand the pathophysiology of leukaemia cells in human bone marrow. Materials and Methods: Six human leukaemia cell lines were cultured under normoxic or hypoxic conditions. Cell growth, recovery of clonogenic cells, and the expression and activation of various signalling proteins were examined. Results: Hypoxia suppressed cell growth and the recovery of clonogenic cells. Moreover, hypoxia up-regulated hypoxia-inducible factor (HIF) $1 \alpha$ and HIF $2 \alpha$ expression while suppressing the expression and activation of NOTCH1, mechanistic target of rapamycin kinase (mTOR) activation, and nuclear factorkappa B (NF-kB) phosphorylation. Conclusion: We found that hypoxia up-regulated HIF expression while it suppressed the self-renewal capacity of leukaemia cells, NOTCH activity, and expression of its down-stream signalling molecules, which differs from previous reports mentioning that HIF activates NOTCH signalling. Our findings serve to further elucidate the in vivo pathophysiology of leukaemia cells.
\end{abstract}

Leukaemia stem cells exist under hypoxic conditions in human bone marrow. In order to better understand the pathophysiology of leukaemia stem cells, it is therefore, important to investigate the signalling pathways associated with a hypoxic environment. Hypoxia is known to stabilize hypoxia-inducible factor (HIF), a DNA-binding transcription factor, which transactivates various genes to adapt to hypoxic conditions. HIFs are heterodimers comprising an oxygenlabile $\alpha$-subunit (HIF $\alpha$ ) and a constitutively expressed $\beta$ subunit (HIF $\beta)$ (1). The HIF family has three members, HIF1, HIF2, and HIF3. HIF1-mediated signalling is

Correspondence to: Shuji Tohda, MD, Ph.D., Department of Laboratory Medicine, Tokyo Medical and Dental University, Yushima 1-5-45, Bunkyo-Ku, Tokyo 113-8519, Japan. Tel: +81 358035334, Fax: +81 358035629, e-mail: tohda.mlab@tmd.ac.jp

Key Words: HIF, hypoxia, leukaemia, NOTCH. important for maintenance of both normal hematopoietic stem cells and leukaemia stem cells under hypoxic conditions $(2,3)$. Moreover, HIF1 $\alpha$ was shown to be overexpressed in specific human cancer types, including leukaemia cells $(4,5)$.

NOTCH is a transmembrane receptor, which regulates the stemness of normal hematopoietic cells and leukaemia cells, as we reported previously (6). Two mechanisms are reported to be associated with crosstalk between HIF and NOTCH. The first involves HIF $\alpha$ binding to cleaved NOTCH1, which results in stabilization and activation of the NOTCH signalling pathway (7). The second describes HIF $\alpha$ as a repressor of the NOTCH1-hes family bHLH transcription factor 1 (HES1) negative feedback loop by inhibiting the binding of HES1 to the HES1 promoter, resulting in enhanced NOTCH signalling (8). However, the precise relationship between HIF and NOTCH has not been determined, specifically, in leukaemia cells.

In order to better understand the influence of hypoxia on the growth of leukaemia cells, we examined the effects of hypoxia on the capacity for self-renewal, which was evaluated using the recovery rate of clonogenic cells in methylcellulose media following suspension culturing. We also examined the influence of hypoxia on the expression and activity of various signalling proteins including HIF, $\mathrm{NOTCH}$, mechanistic target of rapamycin kinase (mTOR), and nuclear factor-kappa B (NF-kB) in leukaemia cells.

\section{Materials and Methods}

Cell lines. Three human acute myeloid leukaemia cell lines (TMD7, THP-1, and NB4), two T-acute lymphoblastic leukaemia cell lines (KOPT-K1 and DND-41), and one chronic myelogenous leukaemia cell line (K562) were used. The TMD7 cell line was established in our laboratory (9), whereas the THP-1 and K562 cell lines were obtained from the Health Science Research Resource Bank (Osaka, Japan). The NB4 cell line was kindly provided by Dr. M. Lanotte (Paris, France), and the KOPT-K1 and DND-41 cell lines were gifted by Drs. Harashima and Orita (Fujisaki Cell Center, Japan). Cell growth assay. Short-term growth was evaluated using a colourimetric assay kit (Dojindo Laboratories, Kumamoto, Japan). 


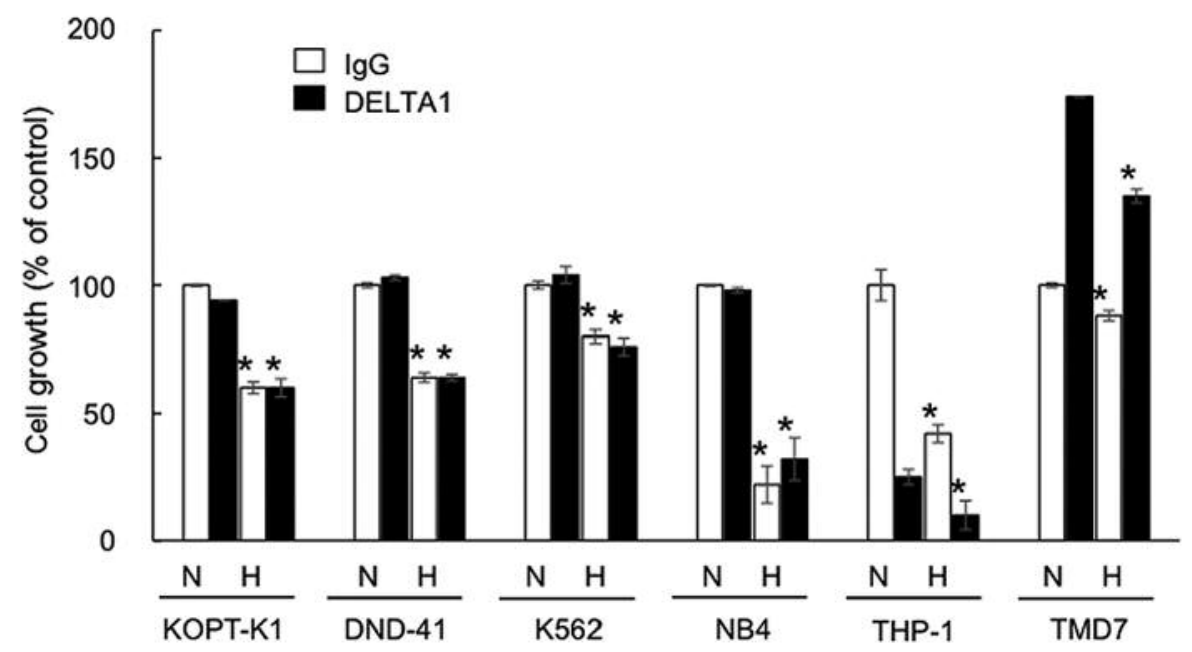

Figure 1. Effects of hypoxia $(H)$ on short-term growth of leukaemia cells cultured with and without NOTCH ligand DELTA1. After 4 days culture with DELTA1 or IgG as a control, cell growth was examined using a colourimetric assay. Growth is shown as the percentage of the mean optical density value normalized to that of cells cultured with $\operatorname{Ig} G$ under normoxia $(N)$. *Statistically significantly different from growth under normoxic conditions $(p<0.05)$.

Cells were cultured in 96-well plates in RPMI-1640 media supplemented with $10 \%$ foetal bovine serum (FBS) under normoxia or hypoxia $\left(1 \% \mathrm{O}_{2}\right)$ in a MULTIGAS incubator (Juji Field Inc., Tokyo, Japan). After 4 days, the tetrazolium salt, WST-8, was added and the optical density (OD) was measured using an enzyme-linked immunosorbent assay plate reader to determine the relative cell number. Student's $t$-test was performed to determine statistical significance of differences. To examine the influence of $\mathrm{NOTCH}$ activation, cells were cultured in plates coated with recombinant NOTCH ligand DELTA1 protein fused to a human IgG1-Fc (gifted from Dr. S. Sakano, Asahi Kasei Corporation, Japan), or IgG1-Fc as a control, as previously described (9).

Self-renewal capacity. The self-renewal capacity of leukaemia cells was evaluated by examining the recovery of clonogenic cells after suspension culture, as described previously (10). Cells were cultured in $1 \mathrm{ml}$ of culture media in 24 -well plates under normoxia or hypoxia $\left(1 \% \mathrm{O}_{2}\right)$. After 2 days, cells were harvested, and the cells were counted. The harvested cells were then plated in $0.1 \mathrm{ml}$ of methylcellulose media in 96-well plates under normoxia. After 4 days, the colonies were counted. The plating efficiency following suspension culturing $\left(\mathrm{PE}_{\mathrm{s}}\right)$ was calculated as the mean colony number divided by the number of cells plated. The clonogenic cells recovered (CCR) were calculated by multiplying the number of harvested cells by the $\mathrm{PE}_{\mathrm{s}}$. Thus, the CCR represents the selfrenewal capacity of leukaemia cells.

Immunoblot analysis. The lysates from the cells cultured under normoxia or hypoxia were subjected to sodium dodecyl sulphatepolyacrylamide gel electrophoresis and analysed by immunoblotting with antibodies against various signalling proteins, including HIF1 $\alpha$, HIF1 $\beta$, NOTCH, cleaved NOTCH1, HES1, Myc protooncogene (MYC), phosphatase and tensin homolog (PTEN), mTOR, phosphorylated (p) -mTOR, protein kinase B (AKT), p-AKT, ribosomal protein S6 kinase (S6K), p-S6K, eukaryotic translation initiation factor 4E-binding protein 1 (4E-BP1), p-4E-BP1, NF-kB (p65) and p-NF-kB (p65) (Cell Signaling Technology, Danvers, MA, USA), and HIF2 $\alpha$ (Novus Biologicals, Centennial, CO, USA); $\alpha$-tubulin (Abcam, Cambridge, MA, USA) was employed as a loading control. Immunoreactive bands were detected using a Pierce Enhanced Chemiluminescent Western Blotting Substrate (Pierce Biotechnology, Rockford, IL, USA). Each assay was repeated a minimum of three times to ascertain reproducibility.

Microarray analysis. To assess the transcriptional effects of hypoxia, changes in mRNA expression were examined via microarray analysis. THP-1 and TMD7 cell lines were cultured under hypoxia or normoxia for $24 \mathrm{~h}$. Total RNA was extracted using a High Pure RNA Isolation kit (Roche Diagnostics, Mannheim, Germany). RNA was then used to prepare cyanine-3-labelled cRNA which was hybridized to a SurePrint G3 Human GE microarray (Agilent Technologies, Santa Clara, CA, USA). The expression profile was analysed using the Agilent Feature Extraction 10.7.1.1. software (Agilent Technologies).

\section{Results}

Effects of hypoxia on short-term growth and self-renewal capacity. Figure 1 shows the effect that hypoxia has on the short-term growth of cells cultured with or without DELTA1 stimulation. Results revealed that hypoxic conditions significantly reduced the growth of all cell lines, compared to those grown under normoxic conditions. Moreover, DELTA1 stimulation acted to suppress the growth of THP-1 cells while promoting that of TMD7 cells under normoxia, as we previously reported (11). Furthermore, hypoxic conditions were not seen to alter the DELTA1-induced growth changes in these two cell lines. However, in the other four cell lines, DELTA1 stimulation did not significantly affect the growth irrespective of normoxia or hypoxia. 
Table I. Effects of hypoxia on clonogenic cells recovered (CCR) after suspension culture. CCR value was calculated by multiplying the number of cells harvested after suspension culture (cell number) by the plating efficiency in methylcellulose media after suspension culture (PE $E_{s}$.

\begin{tabular}{lcccccc}
\hline Cell line & \multicolumn{2}{c}{ Cell no. $\left(\times 10^{5}\right)$} & \multicolumn{2}{c}{$\mathrm{PE}_{\mathrm{S}}\left(\times 10^{-2}\right)$} & \multicolumn{2}{c}{$\mathrm{CCR}\left(\times 10^{4}\right)$} \\
\cline { 2 - 7 } & Normoxia & Hypoxia & Normoxia & Hypoxia & Normoxia & Hypoxia \\
\hline K562 & 1.3 & 1.4 & 66.8 & $13.7^{*}$ & 8.7 & $1.9^{*}$ \\
NB4 & 5.7 & 3.2 & 15.3 & $3.7^{*}$ & 8.7 & $1.2^{*}$ \\
THP-1 & 4.8 & 3.5 & 11.1 & $6.3^{*}$ & 5.3 & $2.2^{*}$ \\
\hline
\end{tabular}

*Statistically significant difference from that under normoxic conditions $(p<0.05)$.

As shown in Table I, hypoxia significantly reduced the number of CCR in three of the examined cell lines compared to those under normoxia. This suggests that hypoxia reduces the self-renewal capacity of leukaemic clonogenic cells under this in vitro experimental condition.

Effect of hypoxia on HIF expression and DELTA1-induced NOTCH activation. Hypoxia was seen to enhance HIF1 $\alpha$ expression in THP-1 and TMD7 cells and HIF2 $\alpha$ expression in THP-1 cells (Figure 2). Moreover, DELTA1 stimulation induced cleavage of NOTCH1 and subsequent up-regulation of HES1 expression. However, hypoxia reduced this DELTA1-induced cleavage of NOTCH1, thereby reducing the up-regulation of HES1 expression in both cell lines.

Effect of hypoxia on expression and activation of signalling proteins. Immunoblotting analysis confirmed that hypoxia increased HIF1 $\alpha$ protein expression (Figure 3). Hypoxia also increased HIF2 $\alpha$ in all experimental cell lines save for NB4 and TMD7 cells. Moreover, hypoxia was seen to reduce the level of NOTCH1 and HES1 in KOPT-K1, K562, and NB4 cell lines. Hypoxia also suppressed MYC expression in all cell lines save for THP-1 cells. Furthermore, hypoxia promoted the phosphorylation of AKT in KOPT-K1, K562, NB4 and THP-1 cells, whereas it functioned to suppress phosphorylation in DND-41 cells. Phosphorylation of mTOR was also promoted in KOPT-K1 and THP-1 cells; however, it was suppressed in DND-41, K562, NB4, and TMD7 cell lines. Hypoxia further suppressed the phosphorylation of S6K and 4E-BP1, which are downstream molecules in the mTOR signalling pathway, in all cell lines. Lastly, hypoxia was found to suppress the phosphorylation of NF- $\mathrm{KB}$ (p65) protein without affecting the level of NF-KB (p65) protein in four cell lines, namely DND-41, KOPT-K1, NB4, and TMD7 cells.

Microarray analysis. Throughout the microarray geneexpression analysis, we focused on target genes associated with the signalling pathways described above. Table II shows the mRNA $\log 2$ ratios between cells cultured under hypoxia and those cultured under normoxia. Expression of HIF target genes

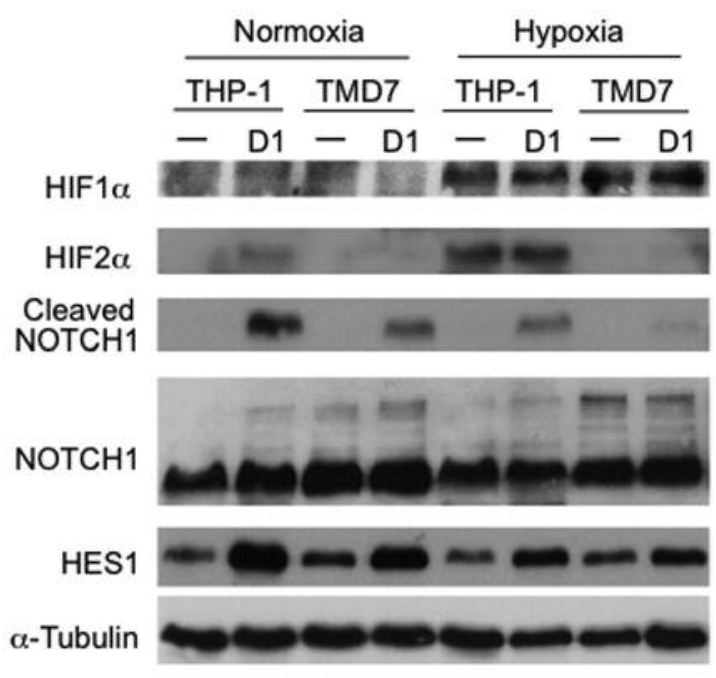

Figure 2. Effect of hypoxia on hypoxia-inducible factor (HIF) expression and DELTA1-induced NOTCH activation. Cells were cultured with or without DELTA1 stimulation under normoxia and hypoxia for $24 \mathrm{~h}$. The lysates from the harvested cells were analysed by immunoblotting. D1: cells stimulated with DELTA1, HES1: Hes family bHLH transcription factor 1 .

were observed to be up-regulated following culturing in hypoxia in both THP-1 and TMD7 cells, which suggests that HIF signalling was activated by hypoxia. Additionally, the expression of NF-kB target genes were found to be downregulated following culturing of TMD7 under hypoxic conditions, which agrees with the results of the immunoblot analysis.

\section{Discussion}

Our initial results showed that hypoxia suppressed the shortterm growth of leukaemia cells (Figure 1). We, therefore, hypothesized that the hypoxia-induced suppression may be caused by leukaemia cells entering into a dormant state similar to that of stem/progenitor cells. This was the rationale for quantifying the CCR under hypoxic conditions. 


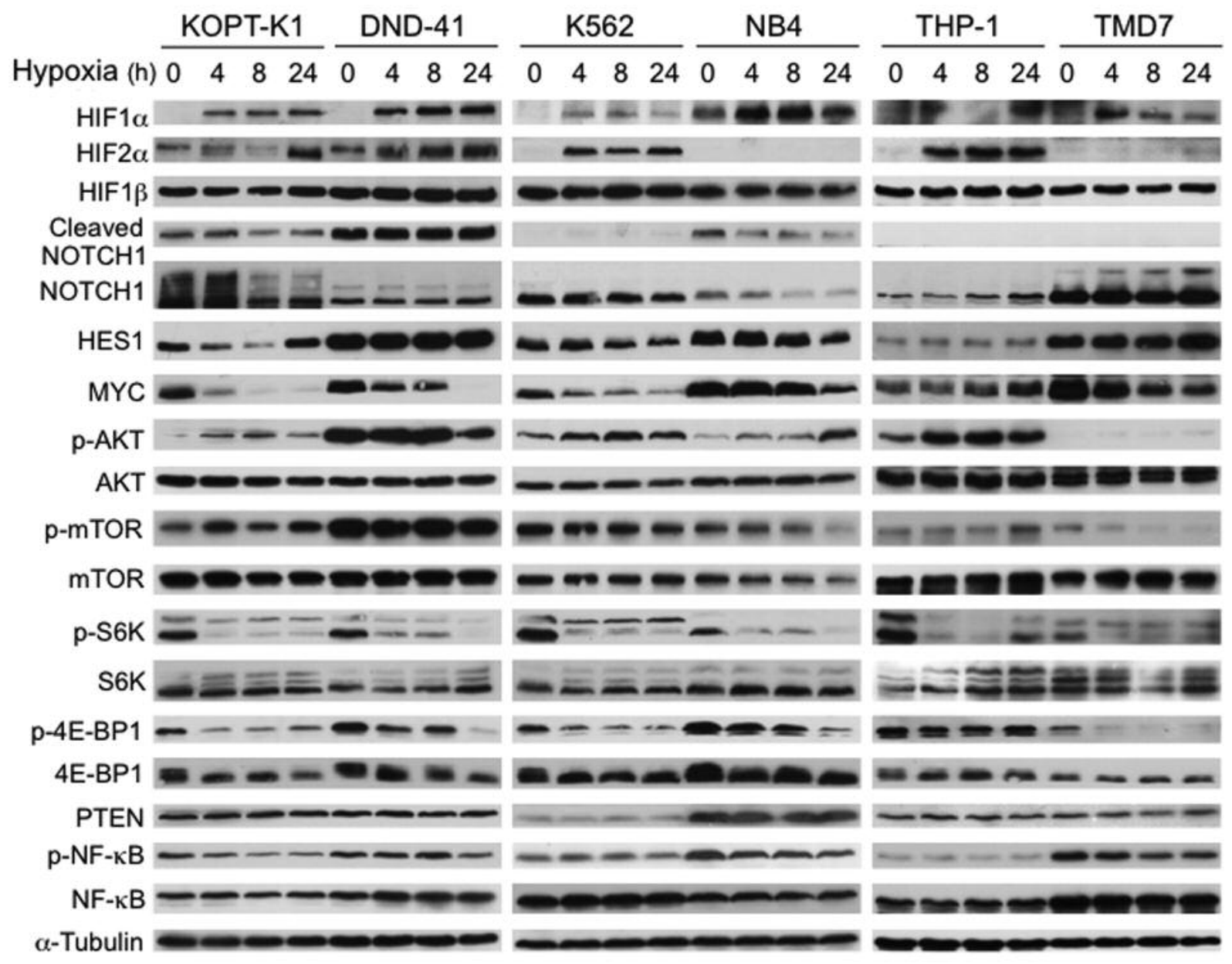

Figure 3. Effect of hypoxia on the expression and activation of various signalling proteins. Cells were cultured under hypoxia for the indicated duration. The lysates from the harvested cells were analysed by immunoblotting. HIF: Hypoxia-inducible factor, HES1: Hes family bHLH transcription factor 1, AKT: protein kinase B, mTOR: mechanistic target of rapamycin kinase, S6K: ribosomal protein S6 kinase, 4 E-BP1: eukaryotic translation initiation factor 4E-binding protein 1, PTEN: phosphatase and tensin homolog, NF-kB: nuclear factor-kappa B, p: phosphorylated.

However, the results indicating that hypoxia suppressed the number of CCR, refuted this hypothesis (Table I). We had also postulated that hypoxia may augment the growthmodulatory effect induced by NOTCH activation in THP-1 and TMD7 cells. However, hypoxia was not seen to affect NOTCH-induced growth suppression of THP-1 cells obviously, nor did it influence growth promotion of TMD7 cells (Figure 1).

We next examined the relationship between hypoxia and NOTCH under our experimental conditions because it has been reported that HIF stimulates NOTCH signalling $(7,8)$. Our results revealed a novel relationship between hypoxia and NOTCH signalling, which disagrees with previously reported mechanisms (Figures 2 and 3). Nevertheless, our findings suggest that hypoxia suppressed NOTCH1 activation, while also acting to increase the expression of
HIF $1 \alpha$ and HIF2 $\alpha$ in specific cell lines (Figure 4). Although we do not have definitive evidence that up-regulation of HIF directly suppressed NOTCH activity, we did clearly demonstrate that up-regulation of HIF does not consistently lead to NOTCH activation.

Hypoxia was also found to suppress MYC expression, which is likely due, in part, to NOTCH suppression (6). Moreover, the effect that hypoxia had on the phosphorylation of AKT and mTOR varied depending on the specific cell line; while hypoxia did consistently suppress phosphorylation of S6K and 4E-BP1, which would result in suppressed protein synthesis. Hypoxia also reduced the activation NF-kB (p65), which may, in part, be related to NOTCH suppression (12). Although the precise relationship between HIF, NOTCH, mTOR, MYC, and NF-kB is very complex and dynamic in each cell line, our results provide evidence that hypoxia-induced suppression of these 


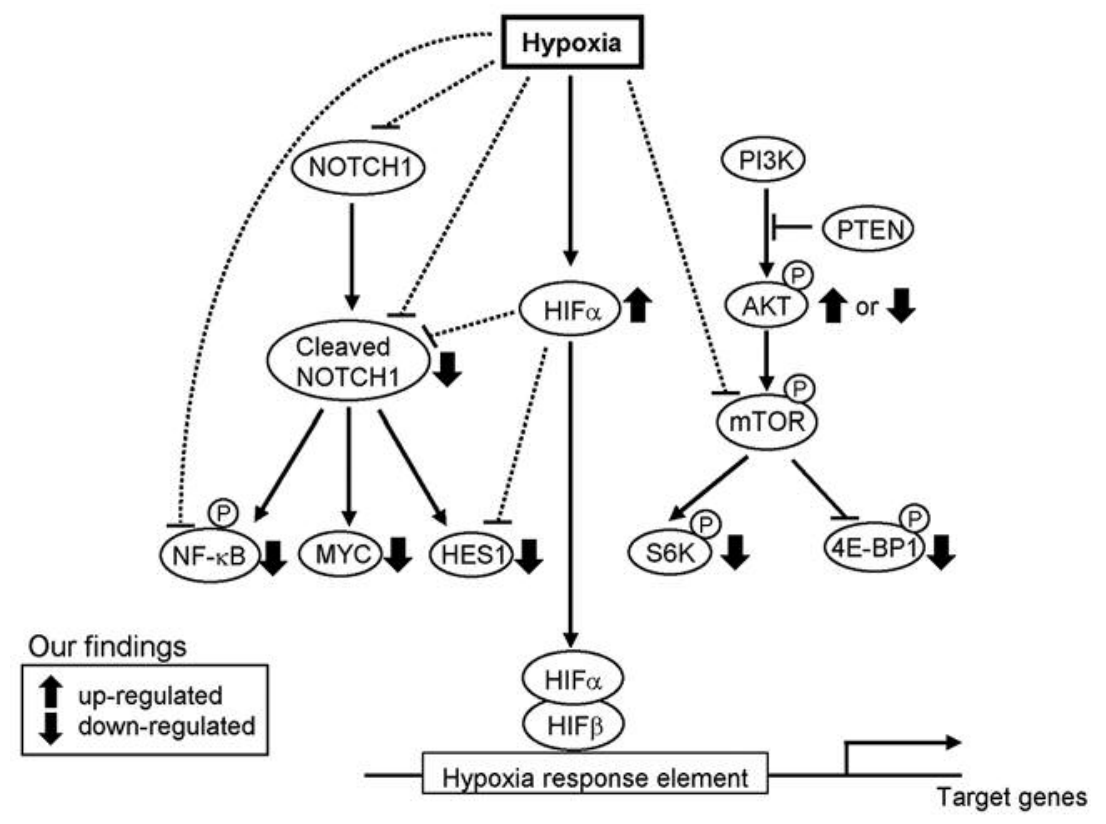

Figure 4. Schematic representation of effects of hypoxia on the activity of signalling proteins. Dotted lines represent the approximate results obtained in this study although the direct causal relationship is not shown. HIF: Hypoxia-inducible factor, NF-kB: nuclear factor-kappa B, HES1: Hes family bHLH transcription factor 1, PI3K: phosphatidylinositol-3 kinase, PTEN: phosphatase and tensin homolog, AKT: protein kinase B, mTOR: mechanistic target of rapamycin kinase, S6K: ribosomal protein S6 kinase, 4E-BP1: eukaryotic translation initiation factor 4E-binding protein 1, P: phosphorylated.

Table II. Effects of hypoxia on the expression of representative target genes for signalling pathways. Expression of mRNA was examined by microarrays. Numbers indicate log2 ratios of mRNA expression in cells cultured under hypoxia for 24 h normalized to that of control cells cultured under normoxia.

\begin{tabular}{lcccc}
\hline Signalling pathway & Target gene & Encoded protein & THP-1 & TMD7 \\
\hline NF-kB & $I L 1 B$ & Interleukin 1 beta & 1.882 & -0.836 \\
& $T N F$ & Tumor necrosis factor & 0.125 & -1.019 \\
NOTCH & BCL2 & B-Cell lymphoma 2 & 0.163 & -0.429 \\
HIF & MYC & Myc proto-oncogene & 1.485 & -1.162 \\
& GLUT1 & Glucose transporter 1 & 0.991 & 1.189 \\
AKT & LDHA & Lactate dehydrogenase A & 2.360 & 1.266 \\
& VEGFA & Vascular endothelial growth factor A & 0.815 & 2.469 \\
& CDN1 & Cyclin D1 & -0.170 & -0.995 \\
\hline
\end{tabular}

NF-kB: Nuclear factor-kappa B; HIF: hypoxia-inducible factor; AKT: protein kinase B.

signalling molecules is partially responsible for the suppression of cell growth and self-renewal capacity.

In this study, we demonstrated a role for hypoxia in the regulation of cell growth and the activity of various signalling molecules. Although cell culture experiments are generally performed under normoxic conditions. The results obtained from experiments under normoxia may, therefore, differ from the phenomena observed in patients' bone marrow. We, therefore, decided to employ hypoxic conditions to mimic leukaemia cells residing in bone marrow. Although our experimental conditions did not necessarily reproduce the conditions in human bone marrow exactly, as our culture system lacked stromal cells, our findings will nevertheless contribute to the development of novel molecular-targeted therapies against leukaemia, specifically for leukaemia stem cells under hypoxic conditions. 


\section{Conflicts of Interest}

The Authors have no conflict of interests to declare.

\section{Authors' Contributions}

MI and ST initiated and designed the study. MI, YO, and ES performed the cell culture, western blotting, and mRNA expression experiments. MI, YT, YS, SM, TS, and ST analysed and interpreted the data. MI and ST wrote and revised the article.

\section{Acknowledgements}

This study was supported in part by JSPS KAKENHI Grant Number C: 17K09005.

\section{References}

1 Wielockx B, Grinenko T, Mirtschink P and Chavakis T: Hypoxia pathway proteins in normal and malignant hematopoiesis. Cells 8: E155, 2019. DOI: 10.3390/cells8020155

2 Takubo K and Suda T: Roles of the hypoxia response system in hematopoietic and leukemic stem cells. Int J Hematol 95: 478483, 2012. PMID: 22539363. DOI: 10.1007/s12185-012-1071-4

3 Zhang H, Li H, Xi HS and Li S: HIF $1 \alpha$ is required for survival maintenance of chronic myeloid leukemia stem cells. Blood 119: 2595-2607, 2012. PMID: 22275380. DOI: 10.1182/blood-201110-387381

4 Keith B, Johnson RS and Simon MC: HIF1 $\alpha$ and HIF2 $\alpha$ : Sibling rivalry in hypoxic tumour growth and progression. Nat Rev Cancer 12: 9-22, 2011. PMID: 22169972. DOI: $10.1038 / \mathrm{nrc} 3183$

5 Giambra V, Jenkins C, Lam SH, Hoofd C, Belmonte M, Wang X, Gusscott S, Gracias D and Weng AP: Leukemia stem cells in TALL require active HIF $1 \alpha$ and WNT signaling. Blood 125: 39173927, 2015. PMID: 25934477. DOI: 0.1182/blood-2014-10-609370
6 Tohda S: NOTCH signaling roles in acute myeloid leukemia cell growth and interaction with other stemness-related signals. Anticancer Res 34: 6259-6264, 2014. PMID: 25368222.

7 Gustafsson MV, Zheng X, Pereira T, Gradin K, Jin S, Lundkvist J, Ruas JL, Poellinger L, Lendahl U and Bondesson M: Hypoxia requires NOTCH signaling to maintain the undifferentiated cell state. Dev Cell 9: 617-628, 2005. DOI: 10.1016/j.devcel.2005. 09.010

8 Wang Y, Liu Y, Malek SN, Zheng P and Liu Y: Targeting HIF1 $\alpha$ eliminates cancer stem cells in hematological malignancies. Cell Stem Cell 8: 399-411, 2011. DOI: 10.1016/j.stem.2011.02.006

9 Tohda S, Sakano S, Ohsawa M, Murakami N and Nara N: A novel cell line derived from de novo acute myeloblastic leukaemia with trilineage myelodysplasia which proliferates in response to a NOTCH ligand, DELTA-1 protein. Br J Haematol 117: 373-378, 2002. PMID: 11972520. DOI: 10.1046/j.1365-2141. 2002.03446x

10 Tohda S, Yang GS, Ashman LK, McCulloch EA and Minden MD: Relationship between c-KIT expression and proliferation in acute myeloblastic leukemia cell lines. J Cell Physiol 154: 410-418, 1993. PMID: 7678840. DOI: $10.1002 /$ jcp. 1041540225

11 Murata-Ohsawa M, Tohda S and Nara N: Cellular analysis of growth suppression induced by NOTCH ligands, DELTA-1 and JAGGED-1 in two myeloid leukemia cell lines. Int J Mol Med 14: 223-226, 2004. PMID: 15254769. DOI: 10.3892/ ijmm.14.2.223

12 Itoh $\mathrm{M}, \mathrm{Fu} \mathrm{L}$ and Tohda S: NF-kB activation induced by NOTCH ligand stimulation in acute myeloid leukemia cells. Oncol Rep 22: 631-634, 2009. PMID: 19639214. DOI: 10.3892/or_00000482

Received May 22, 2019

Revised June 18, 2019

Accepted June 20, 2019 